\title{
NOTAS
}

\section{EL DEBILITAMIENTO DE /T / EN DOS VARIEDADES DEL ESPAÑOL AMERICANO}

La realización debilitada de la africada / $\mathrm{t} \int /$ essin duda un fenómeno de gran vitalidad en el español americano. Se trata de un proceso ampliamente extendido en la región del noroeste mexicano que comprende el norte de Nayarit, el noroeste de Durango, Sonora, Chihuahua, Baja California'; en el español de Sonora constituye uno de los rasgos característicos que pervive incluso en hablantes que han cambiado de lugar de residencia2. Más allá de las fronteras mexicanas, este proceso se ha reportado, como fenómeno variable, en algunas variedades del español de Puerto Rico ${ }^{3}$, de Panamá, Cuba y del caribe colombiano ${ }^{4}$.

En este trabajo abordaré el fenómeno en cuestión con datos de dos dialectos americanos: el mexicano, en particular el del habla sonorense, y el panameño.

En el proceso de debilitamiento la africada pierde o reduce la longitud de su momento de oclusión, así, se realiza ya como una fricativa plena, ya como una fricativa precedida por un brevísimo momento de oclusión. El interés que presenta el fenómeno no reside en los contextos en los que ocurre la fricativa plena, sino en el estudio de las secuencias de nasal o líquida homorgánica más fricativa, contextos en los cuales la / $\mathrm{t} \int /$ presenta una breve oclusión $^{5}$.

' José Moreno de Alba, La pronunciación del español en México, El Colegio de México, México, 1994.

2 Dolores Brown, "El habla juvenil de Sonora, México: la fonética de 32 jóvenes", NRFH, 37 (1989), 43-82; FÉlix ANDrÉs AcosTA, "Estado actual de los estudios dialectales y sociolingüísticos del español de Sonora", en Memorias del VEncuentro Internacional de Lingüistica en el Noroeste, eds. M. del C. Morúa y G. López C., Universidad de Sonora, Hermosillo, 2000, t. 2, pp. 247-264.

${ }^{3}$ ANTONio Quilis y MARía VAQUERo, "Realizaciones de la / $\mathrm{ch} /$ en el área metropolitana de San Juan de Puerto Rico", RFE, 56 (1973), 1-52 y Humberto López MoRALES, Estratificación social del español de:San Juan de Puerto Rico, UNAM, México, 1983.

${ }^{4}$ José Joaquín Montes GiRaldo, Dialectología general e hispanoamericana, Publicaciones del Instituto Caro y Cuervo, SantaFé de Bogotá, 1995. NACYT)

5 Esta investigación se realizó en el marco del proyecto U44731-H (SEP-CO- 
Los resultados del estudio instrumental revelan que esa breve oclusión seguida de la fricción no constituye un segmento africado en plenitud, sino que es el resultado de un gesto de transición que posibilita la secuencia de [nasal] [fricativa] y [lateral] [fricativa] homorgánica. A la luz de lo que sucede tanto en la fonología del propio español, como en la de distintas lenguas, es posible suponer que las secuencias, ya de una nasal o una lateral más una fricativa homorgánicas, son secuencias marcadas y como consecuencia tienden a evitarse mediante distintas estrategias.

\section{Metodología}

Los datos de la variante sonorense fueron proporcionados por dos hombres de alrededor de 40 años, originarios y residentes de Hermosillo, ambos con el oficio de albañil y con poca escolaridad. Para la variante panameña se trabajó con una mujer de alrededor de 30 años, estudiante de Maestría en el D.F. ${ }^{6}$ Los datos de Hermosillo se recogieron mediante dos entrevistas de 70 minutos cada una, en ellas les solicité que me hablaran del oficio de albañilería; les leí, además, una pequeña historia y al término les pedí su opinión sobre el contenido; incluí la lectura de una breva lista de palabras; una serie de preguntas en las que les proporcioné la descripción de diferentes objetos o acciones y les pedí que me dieran el nombre o el verbo correspondiente. En el caso de la hablante panameña las palabras con $/ \mathrm{t} \int /$ aparecían en un conjunto de frases simples que le solicité que leyera. Los contextos de aparición incluyen el intervocálico y después de consonante resonante (nasal, líquida y vibrante); se cuidó también que la africada apareciera tanto en posición tónica como postónica. Las grabaciones de los sonorenses se hicieron en formato mini disc y posteriormente se analizaron en el laboratorio de El Colegio de México, utilizando el programa CSL de Kay. Para el español panameño las frases se grabaron directamente en el Kay. Para los dos dialectos se obtuvo un total de 104 palabras con el segmento de estudio, de las cuales 60 fueron distintas y 44 repeticiones de algunas de ellas. (Véase el Apéndice.)

\section{ANÁLISIS ACÚSTICO}

El análisis espectrográfico arrojó los siguientes resultados: entre vocales la africada del sistema estándar del español tiene una realización fricativa en la pronunciación sonorense y panameña, ya se encuentre

${ }^{6}$ Agradezco a los señores Luis Jiménez Granillo y Manuel Mejía, así como a Viviana Hernández por su valiosa ayuda. 
en sílaba tónica o postónica. Lo anterior se aprecia en las Figuras 1 y 2 , correspondientes a trapichey a el muchacho, en las dos variedades de estudio.

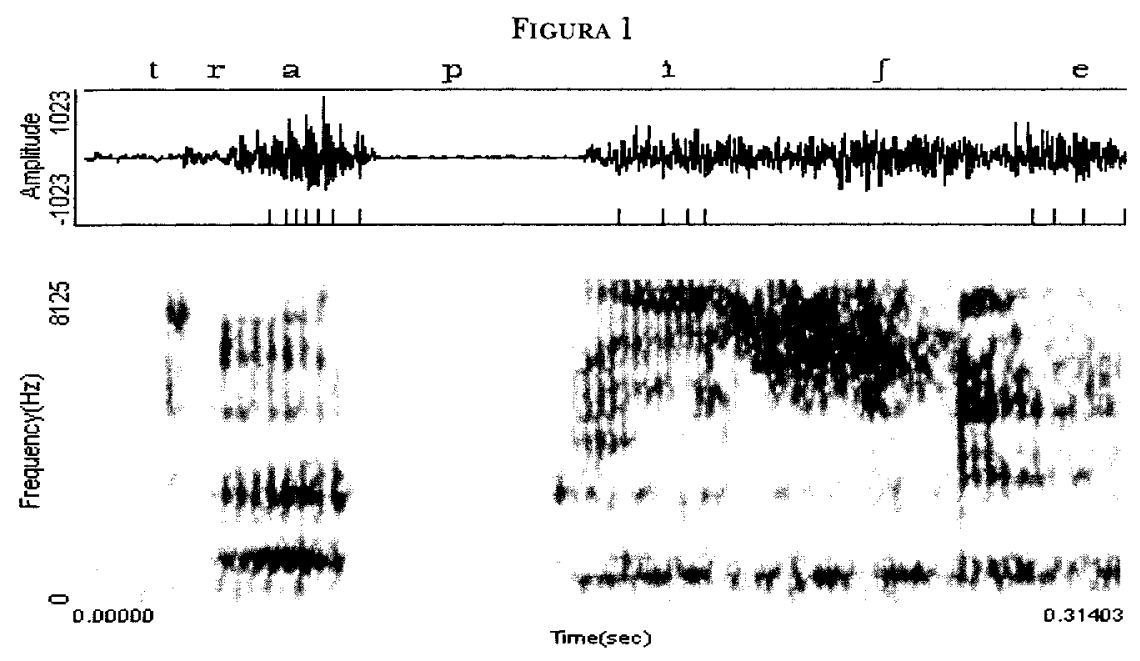

Oscilograma y espectrograma

$\mathrm{de} / \mathrm{t} \int /$ relajada entre vocales (pronunciación sonorense)

Figura 2
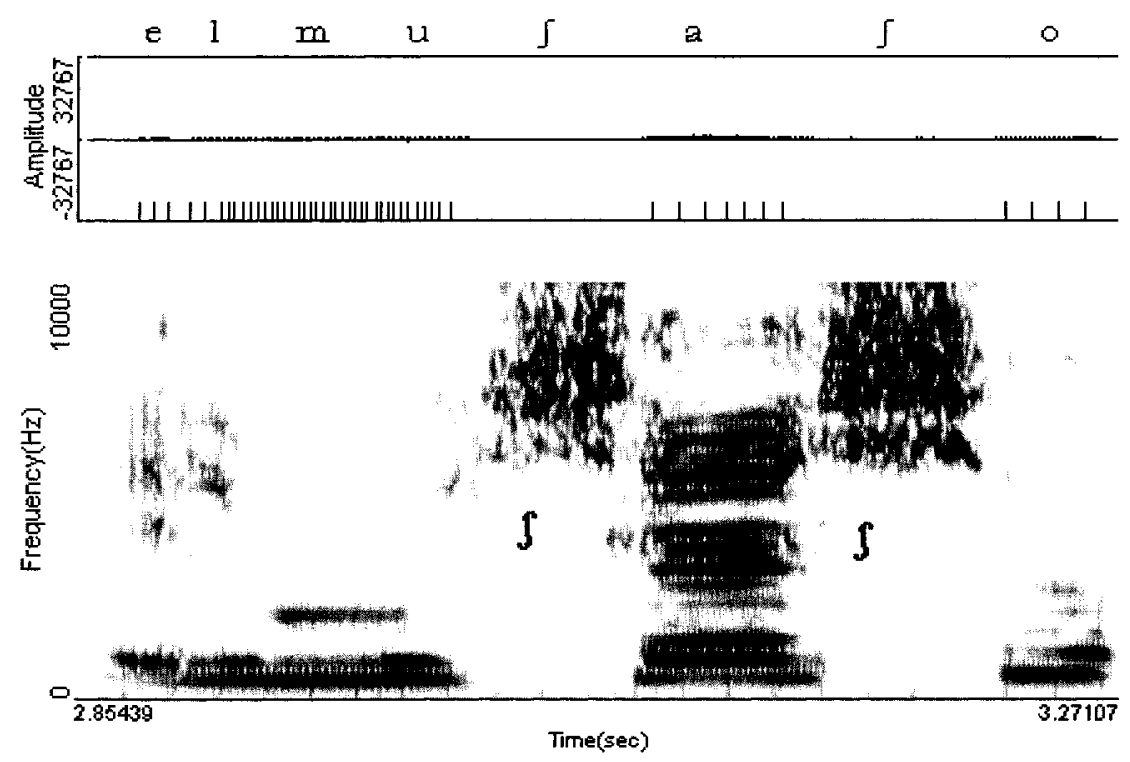

Oscilograma y espectrograma

$\mathrm{de} / \mathrm{t} \int /$ relajada entre vocales (pronunciación panameña) 
Cuando la africada aparece después de consonante nos enfrentamos con la siguiente situación: después de la vibrante se realiza como fricativa. En las Figuras 3 y 4 mostramos el espectrograma de parchey de parchar que ejemplifican nuevamente el contexto postónico y de sílaba tónica.

\section{Figura 3}

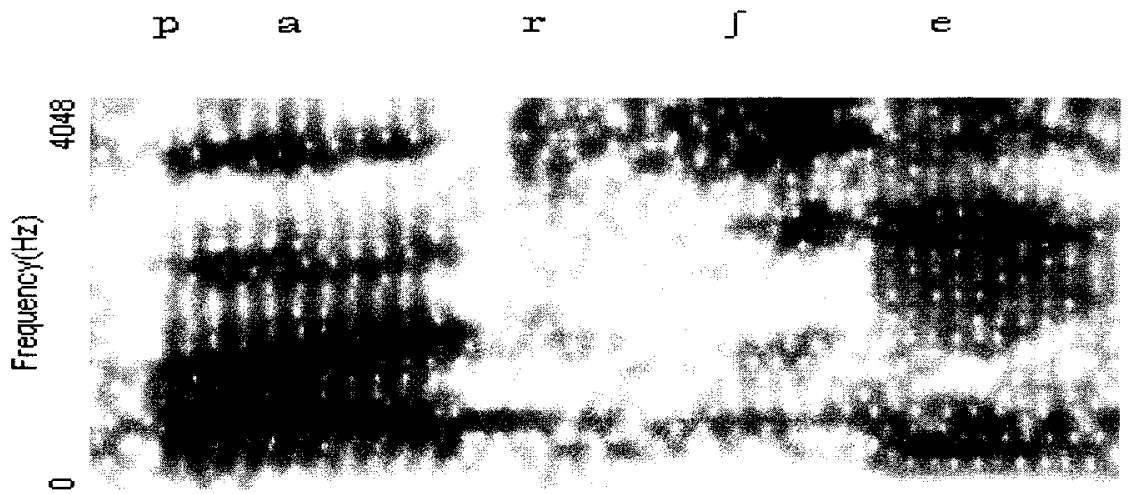

Espectrograma de la realización $\mathrm{de} / \mathrm{t} \int /$ después de vibrante (pronunciación sonorense)

Figura 4

p $\mathrm{a}$

亮

14⿻

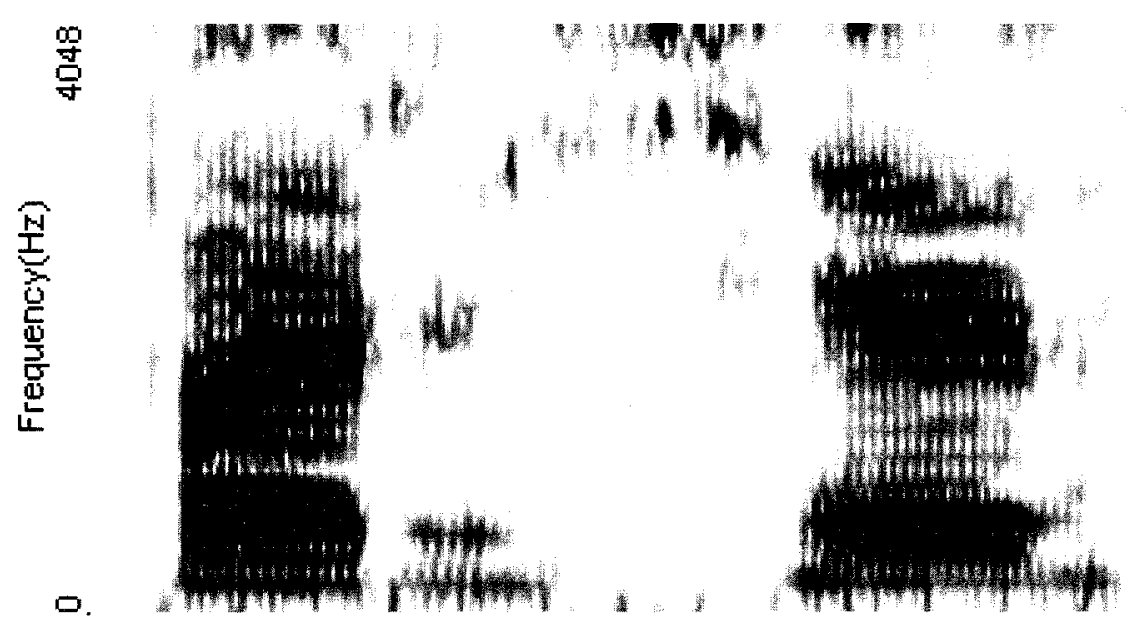

$\mathbf{r}$<smiles>[TlH]</smiles>

a

$r$

Espectrograma de la realización de $/ \mathrm{t} \int /$ después de vibrante (pronunciación panameña) 
El siguiente contexto está representado por la realización de colcha (Figura 5) y tras $/ \mathrm{l} /$ vemos una situación distinta: a la porción fricativa le antecede un momento de cierre, traducido por el silencio y la línea vertical que indica la liberación de aire luego de una oclusión.

Figura 5
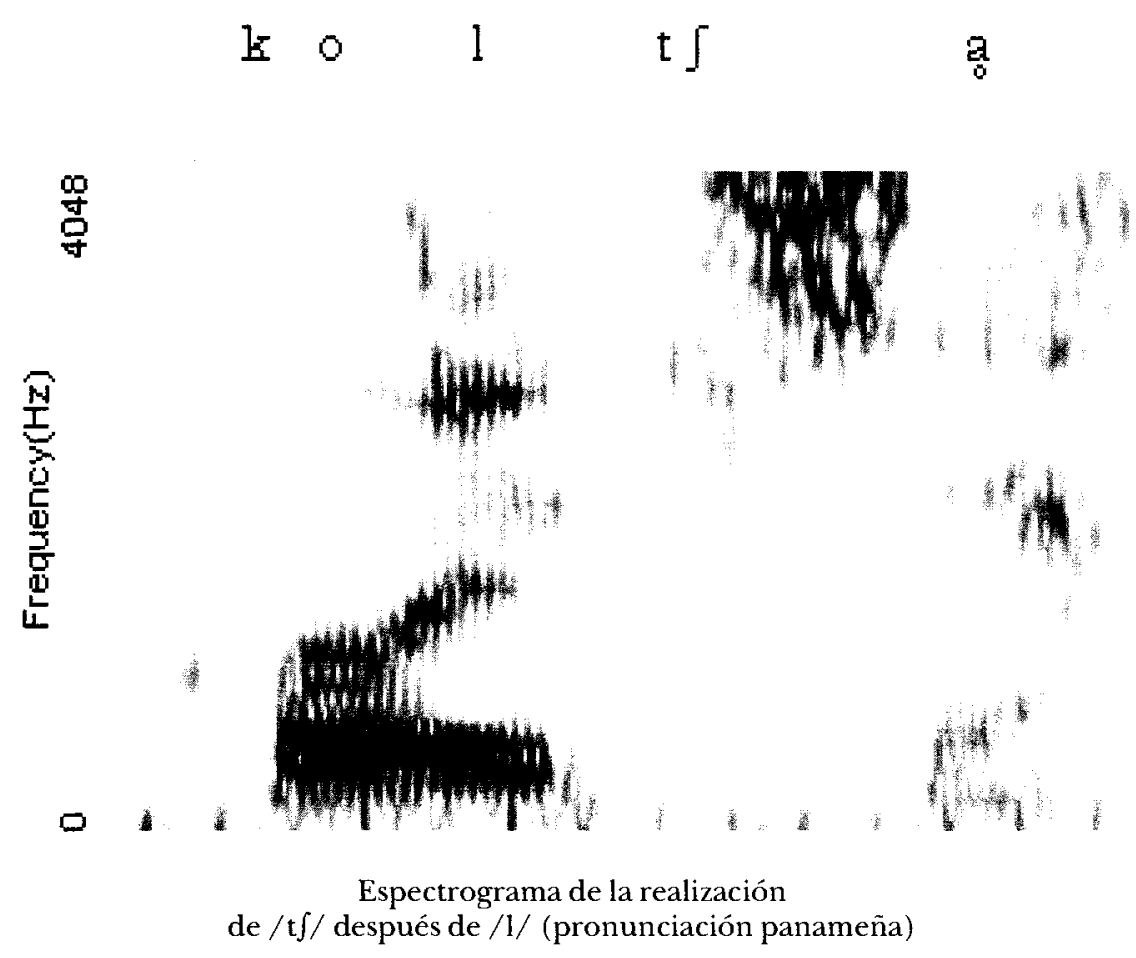

Esta realización de la africada ocurre también cuando media un linde de palabras entre los dos segmentos. La prueba de ello se presenta en la Figura 6; en ella podemos observar que, a diferencia de lo ocurrido en la chavala, en la realización de al chavalo, al igual que en colcha, la africada presenta un momento de oclusión. La Figura 6 también permite ver indicios del punto de articulación de ambas realizaciones. 
Figura 6
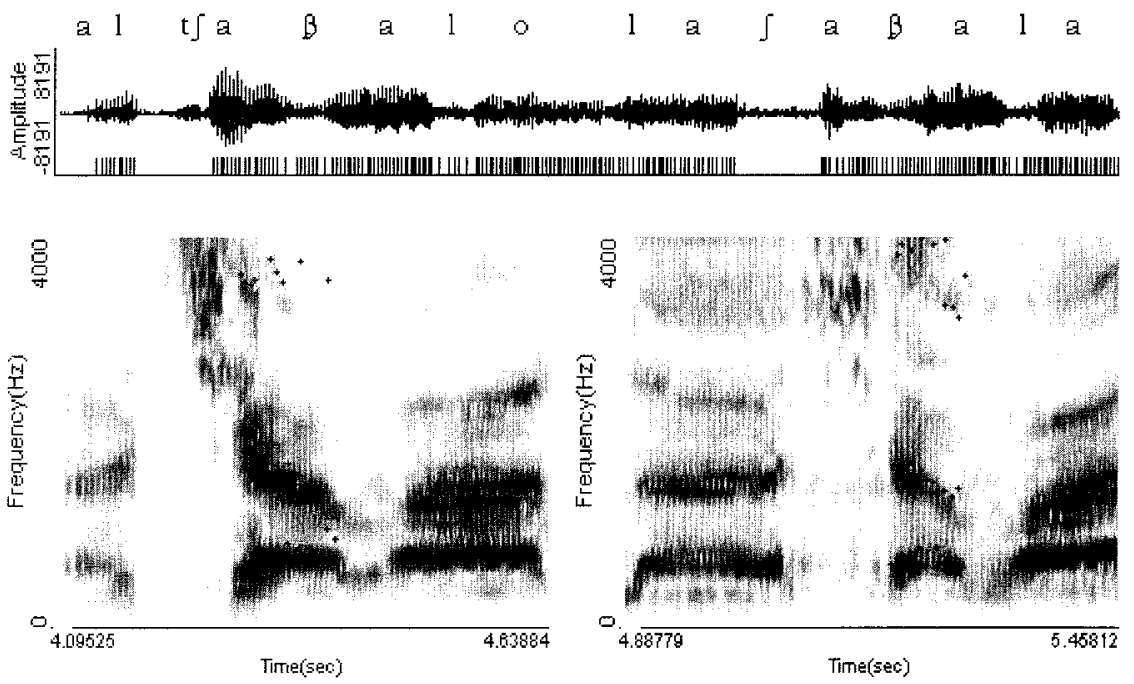

Espectrograma de la alternancia

entre africada y fricativa (pronunciación sonorense)

En las vocales se señala la trayectoria de los formantes de las vocales bajas. $\mathrm{El}_{1}$ tiene una transición negativa en ambos casos; el $\mathrm{F}_{2}$, en cambio, presenta una transición positiva más pronunciada en la vocal [a] después de la $/ \mathrm{t} /$.

Figura 7
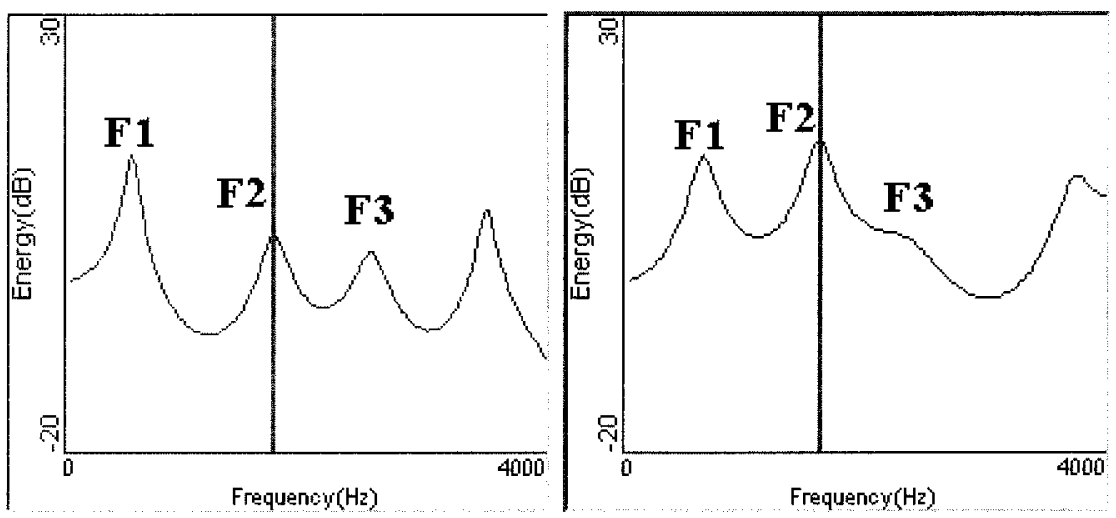

LPC de la ch[a] en "al chavalo"

(izquierda) y de ch[a] en "la chavala"

(pronunciación sonorense) 
La influencia del punto de articulación de la consonante sobre la vocal se puede apreciar mejor en la Figura 7, donde tenemos el LPC de las dos vocales bajas. En la [a] de [alt faßalo] el $\mathrm{F}_{2}$ en la transición se ubica a los 1,705.88 Hz.; mientras que la [a] de [lafaßala] presenta su $\mathrm{F}_{2}$ a los 1,607.84 Hz. Esta diferencia de $100 \mathrm{~Hz}$. sugiere que la africada con una breve oclusión es más palatal que cuando se realiza como fricativa plena.

Por último, la realización de la africada después de nasal se muestra en la Figura 8; contexto en el cual también presenta una breve porción oclusiva.
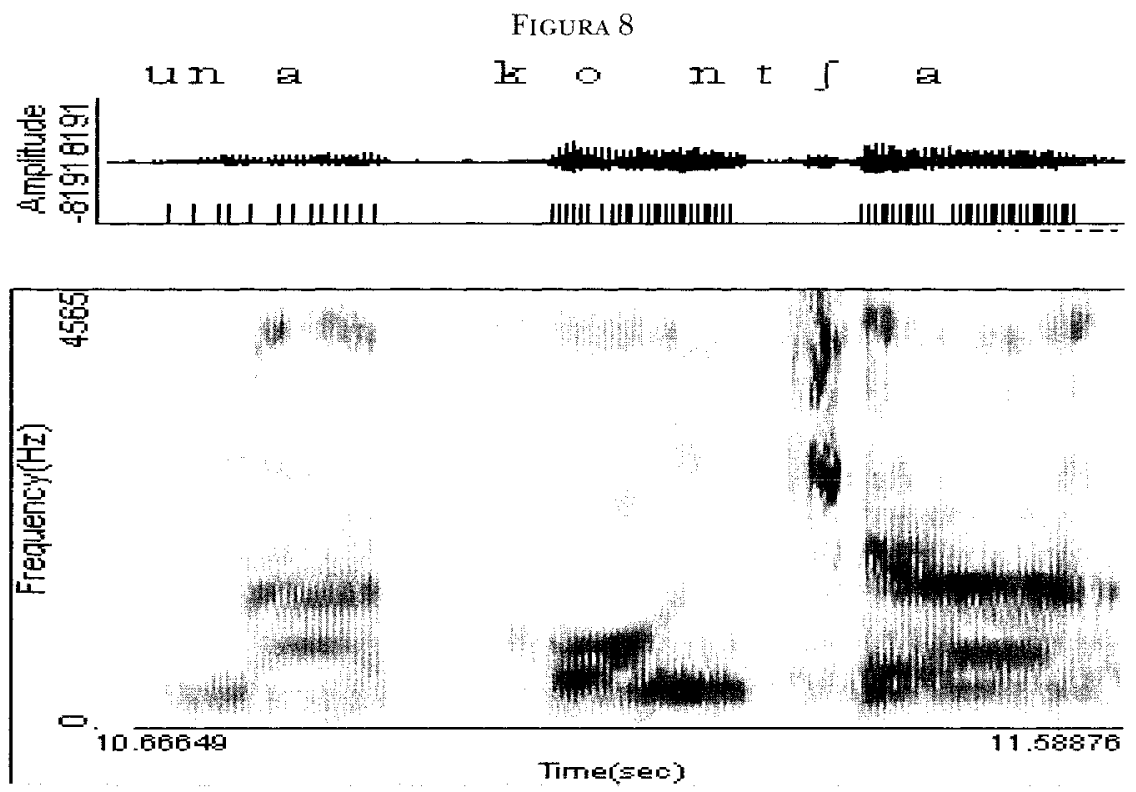

Oscilograma y espectrograma de la realización de $/ \mathrm{t} \int /$ después de consonante nasal (pronunciación sonorense)

Los hechos acústicos muestran que las dos variedades del español presentan resultados similares, en ambas la africada pierde su porción oclusiva cuando se encuentra entre vocales y después de vibrante, sea sílaba tónica o átona, y conserva, por así decirlo, parte de su componente oclusivo cuando se encuentran después de una consonante nasal, o después de una consonante lateral.

Con el fin de determinar el estatus del momento de oclusión se midió su duración y se comparó con la duración de los mismos ítems producidos por hablantes que no presentan el proceso, para ello se tomó a dos hablantes de la Ciudad de México. La Tabla 1 muestra los promedios de duración del cierre y de la fricción en la realización de 
la africada no relajada y de la relajada. El promedio de duración del cierre en la africada objeto de estudio representa sólo $39.11 \%$ en relación con la no relajada.

TABLA 1

Promedio de la duración del cierre y de la fricción

\begin{tabular}{lll}
\hline & tf & $\mathrm{t} \int$ \\
\hline cierre & $29.75 \mathrm{~m} / \mathrm{seg}$. & $76.05 \mathrm{~m} / \mathrm{seg}$. \\
fricción & $74 \mathrm{~m} / \mathrm{seg}$. & $40.45 \mathrm{~m} / \mathrm{seg}$. \\
longitud total & $103 \mathrm{~m} / \mathrm{seg}$. & $116 \mathrm{~m} / \mathrm{seg}$. \\
\hline
\end{tabular}

Los resultados anteriores sugieren que se trata de un gesto de transición que permite el paso de una articulación a otra, impidiendo que una nasal o una líquida se encuentren en contigüidad con una fricativa. Un buen número de procesos parecen confirmar esta hipótesis.

Como primera evidencia mencionaré la sugerente semejanza entre el debilitamiento de la africada y la llamada espirantización de oclusivas en español. Los datos de (1) muestran que la serie de oclusivas / b d g/ se vuelve fricativa entre vocales y después de vibrante, pero después de nasal las tres consonantes conservan lo oclusivo; el comportamiento asimétrico de la líquida indica que ésta asegura el rasgo [-continuo] a la oclusiva sólo si la secuencia es homorgánica:

\section{(1) Alternancia oclusiva-fricativa}

$\begin{array}{llll}\mathrm{V}- & \mathrm{r}- & \mathbf{N} & \mathrm{N}- \\ \mathrm{a}[\boldsymbol{\beta}] \mathrm{a} & \operatorname{cor}[\boldsymbol{\beta}] \mathrm{a} & \operatorname{cal}[\boldsymbol{\beta}] \mathrm{o} & \operatorname{cam}[\mathbf{b}] \mathrm{io} \\ \operatorname{na}[\boldsymbol{\delta}] \mathrm{a} & \operatorname{nar}[\boldsymbol{\delta}] \mathrm{o} & \operatorname{cal}[\mathbf{d}] \mathrm{o} & \operatorname{an}[\mathbf{d}] \mathrm{a} \\ \operatorname{mi}[\boldsymbol{\gamma}] \mathrm{a} & \operatorname{lar}[\boldsymbol{\gamma}] \mathrm{o} & \operatorname{al}[\boldsymbol{\gamma}] \mathrm{o} & \operatorname{hon}[\mathbf{g}] \mathrm{o}\end{array}$

Si estas dos sonorantes no impidieran el debilitamiento tendríamos como resultado sendas secuencias de [nasal] o [líquida] más [fricativa]. La evidencia dice que cuando estas secuencias ocurren las lenguas presentan procesos diversos. Para el español porteño, Rafael Núñez Cedeño y Alfonso Morales Front ${ }^{7}$ reportan que tras una líquida o una nasal, la fricativa [•] se realiza con un breve componente oclusivo, mientras que entre vocales ocurre una fricativa simple. En (2) se muestran algunos datos.

7 Fonología generativa contemporánea de la lengua española, Georgetown University Press, Washington, D.C., 1999, p. 110. 
(2) Alternancia entre []$y\left[^{d}\right]$ en el español porteño ${ }^{8}$

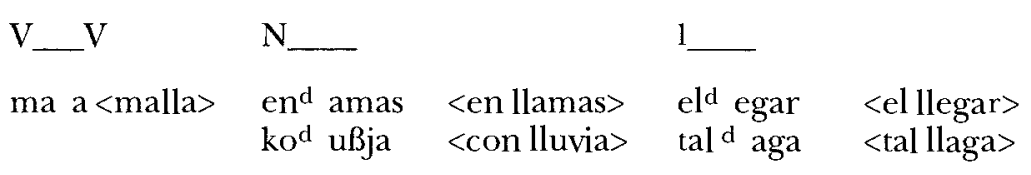

Para el español de México, Raúl Ávila y Juan M. Lope Blanch señalan que a lo largo de la República mexicana hay un fenómeno variable que consiste en un reforzamiento de la /s/ mediante una oclusión ${ }^{9}$. El punto de interés para el tema aquí tratado es qué ocurre después de la nasal y la lateral. Esta situación se ejemplifica con los datos siguientes $^{10}$ :

(3) Reforzamiento de /s/ en el español de México ${ }^{11}$

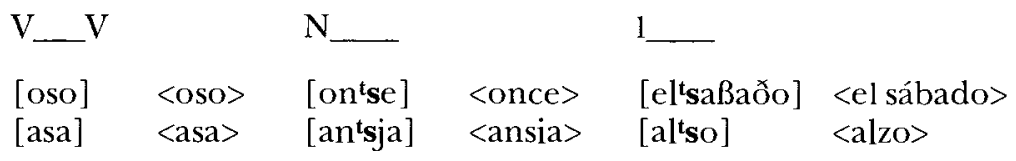

La semejanza con lo que sucede con la africada no es fortuita. De la misma manera que el español porteño y el mexicano evitan esas secuencias marcadas con la intrusión de un breve cierre, así también la africada objeto de estudio presenta una mínima oclusión.

El mixteco, lengua de la familia otomangue, hablada en Guerrero, proporciona una prueba adicional de la alternancia entre fricativas y oclusivas. En esta lengua hay un segmento aproximante bilabial $/ v /$ que se realiza como tal entre vocales, pero se refuerza como oclusiva después de la nasal intrusiva que desarrollan todas las vocales nasales. Así lo podemos apreciar en los datos de (4):

(4) Alternancia [v] [b] en el mixteco de Coscatlán, Guerrero. (El acento grave indica tono bajo, el agudo, el tono alto, el circunflejo, el contorno alto-bajo, el tono medio no se marca) $)^{12}$

$\mathrm{V}$ $\underline{\mathrm{V}}^{\mathrm{N}}$

$/ \mathrm{i}^{\mathrm{n}}$ davi?è $/ \rightarrow\left[\mathrm{i}^{\mathrm{n}}\right.$ davî̀è $]$ "una casa" $/$ tọvî̀è $/ \rightarrow\left[\right.$ too $^{\mathrm{m}}$ bî̀è $]$ "dentro

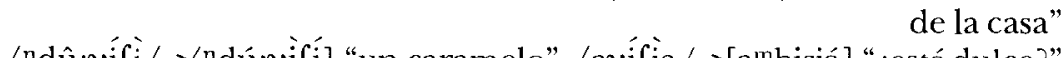

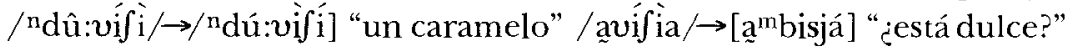

8 Tomado de loc. cit.

9 Raúl Ávila, "Realizaciones tensas de /s/ en la Ciudad de México", $A L M, 11$ (1973), 235-239 y Juan M. Lope Blanch, Ensayos sobre el español de América, UNAM, México, 1993.

${ }^{10}$ Cabe señalar que esta pequeña oclusión no se presenta en palabras como "Alfonso", "delfán"o "alfalfa", en las cuales [1] está seguida por una fricativa heterorgánica.

11 Tomado de R. Ávila, art. cit. yJ. M. LOPE BlANCH, op. cit.

12 Tomado de archivo personal. 
La nasal intrusiva homorgánica entre la vocal nasal asegura el rasgo [- continuo] al segmento siguiente.

Ahora bien, si la secuencia nasal-fricativa se puede resolver mediante la intrusión de una oclusión, o el reforzamiento del segmento siguiente, también hay casos en los que la solución a esa secuencia implica la elisión. Esta situación está representada por otra lengua mexicana, el zoque. En los ejemplos de (5) vemos que el morfema posesivo de tercera persona es un prefijo nasal que asimila su punto de articulación a los segmentos [- continuo] y al tiempo los sonoriza, pero se elide ante consonante fricativa.

(5) Zoque de Chiapas, variante de Chapultenengo ${ }^{13}$

$$
\begin{array}{ll}
N+\text { poki } & \rightarrow \text { mboki "mi rodilla" } \\
N+\text { tahk } & \rightarrow \text { ndthk "mi casa" } \\
N+\text { kak } & \rightarrow \text { ngak "mi tigre" } \\
N+\text { tsuki } & \rightarrow \text { ndzuki "mi tapir" } \\
N+\text { sihs } & \rightarrow \text { sihs "mi carne" } \\
N+\text { Japun } & \rightarrow \text { Japun "mi jabón" }
\end{array}
$$

En suma, tanto la elisión de la nasal, el reforzamiento de la fricativa, así como la intrusión de una oclusión parecen ser estrategias que disuelven las secuencias de nasal o líquida más fricativa. Con base en la evidencia anterior resulta posible plantear que en estas variedades del español americano, el debilitamiento de la africada implica la pérdida del momento de oclusión de / $\mathrm{t} \int / \mathrm{en}$ todos los contextos; la breve oclusión está promovida para impedir que una nasal o una líquida se encuentren en contigüidad con una fricativa.

Respecto a los segmentos nasales, los estudios de corte experimental han mostrado que no ofrece pistas lo suficientemente robustas para vehicular su punto de articulación. El murmullo nasal sólo les asegura la percepción como clase, es decir en su modo de articulación, así lo demuestran los estudios de Malecot, Ohala, Recasén, Kurowsky y Blumstein y Herrera, entre otros ${ }^{14}$. El contexto ideal para

13 Tomado de Esther Herrera Zendejas, Palabras estratos y representaciones: temas de fonología léxica en zoque, El Colegio de México, México, 1995.

14 A. MALECot, "Acoustic cues for nasal consonants: An experimental study involving a tape-splicing technique", $\operatorname{Lan}, 32$ (1956), 274-284; JoHN OHaLA, "Phonetic explanation for nasal sound pattern", en Nasálfest. Papers from a Symposium on nasals and nasalization, eds. C. A. Ferguson, L. M. Hyman \& J. Ohala, Stanford University Press, 1975, pp. 284-316; D. RECAsÉN, "Place cues for nasal consonants with special reference to Catalan", Journal of the Acoustical Society of America, 73 (1983), 1346-1553; K. M. Kurowsky y S. E. Blumstein, "Acoustic properties for perception of nasal consonants", en Phonetics and phonology: Nasal, nasalization and the velum, eds. M. K. Huffman \& R. A. Krakow, Academic Press, San Diego, CA, 1993; y Esther HerreRA ZENDEJAS, "La asimilación de las nasales en español. Un estudio instrumental", NRFH, 50 (2002), 1-14, entre otros. 
que una nasal no empobrezca sus pistas es cuando le precede o le sigue una vocal, o bien cuando le sigue una consonante [- continua]. En la secuencia de nasal fricativa, la fricativa no tiene un momento de brusca soltura que permita a la nasal vehicular su punto de articulación. Los breves momentos de oclusión que median entre la nasal y la fricativa, tanto en el proceso de debilitamiento de la africada, como en las demás lenguas, tienen el efecto de robustecer las pistas de la nasal, permitiendo a la fricativa que preceda una nasal. El caso del zoque muestra que el gesto articulatorio de descenso del velo del paladar no ocurre antes de la fricativa.

A diferencia de una nasal, la líquida es un segmen to cuyo estatus respecto a la continuidad parece variar entre las lenguas. Mientras que en lenguas como el vasco este segmento forma parte de una clase natural con las oclusivas y las nasales ${ }^{15}$, en español, como se mostró con los datos de (1), la líquida es un segmento [+ continuo] al igual que las vocales; la homorganicidad parece ser un requisito para que se comporte como [- continua] y bloquee el proceso de fricativización de las oclusivas ${ }^{16}$. Al respecto, Mascaró ${ }^{17}$ ha esbozado una explicación articulatoria según la cual la líquida actúa como oclusiva con la [d] debido a que durante la producción de [l] no hay corriente de aire en la región dentoalveolar ${ }^{18}$. Esta explicación se puede reforzar para entender el debilitamiento de nuestra africada, agregando que si bien las secuencia [1] [d], así como [l] [fricativa], se articulan en una misma zona, la zona alveolar, en ellas se involucran dos articuladores,

${ }^{15}$ Jeroen VAN de Weljer, "Continuance in liquids and in obstruents", Lingua, 96 (1995), 45-61.

${ }^{16}$ Ibid., p. 47 retoma datos de Hualde del vasco para mostrar que / $\mathrm{t}, \mathrm{n}, \mathrm{l} /$ forman el contex to de elisión de /t/: bat + paratu $\rightarrow$ ba paratu "put one", bat + kurri $\rightarrow$ ba kurri "run one", bat + naka $\rightarrow$ ba naka "one by one", ez + dut Lupe $\rightarrow$ ez du Lupe "I have not seen Lupe". Un caso adicional se encuentra en el pame, lengua otomangue hablada en San Luis Potosí, México. En ella, tanto las nasales como $/ 1 \mathrm{l}^{\mathrm{j}} /$ tienen rea-

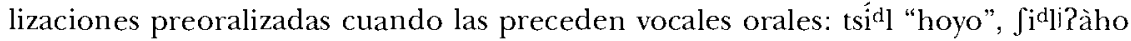
"opinión", talúgn "pollo" (tomado de LoRNA F. GiBson, "Pame [Otomi] phonemics and morphophonemics", IJAL, 4, 1956, 242-265).

${ }^{17}$ Joan Mascaró, "Continuant spreading in Basque, Catalan and Spanish", en Language sound structure, eds. M. Aronoff \& R. Oerle, MIT Press, Cambridge, Mass., pp. 110-121 e "Iberian spirantization and continuant spreading", CatWPL, 1991, núm. 1, 167-179 apud Blanca Palmada, "Continuant spreading and feature organization", en Issues in the phonology and morphology of the major Iberian languages, eds. F. Martinez-Gil \& A. Morales-Front, Georgetown University Press, Washington, D.C., 1997, pp. 151-171.

${ }^{18}$ La noción de prominencia acústica puede agregarse a la explicación de Mascaró. Sabemos que una líquida posee una estructura acústica muy parecida a las vocales (formantes, puede portar tono, ser núcleo silábico y tiene un alto nivel de sonoridad); cuando una [d] se vuelve fricativa presenta una estructura acústica cuasi vocálica. De producirse la fricativización de [d] en ese contexto resultaría una secuencia de segmentos muy semejantes, poniendo en riesgo la posibilidad de que [l] y [d] contrasten. 
para la líquida el articulador activo es el ápice y para la oclusiva y la fricativa es la lámina de la lengua. Entre estos dos gestos articulatorios no hay traslape, sino conflicto articulatorio, mismo que se resuelve, en el caso de [1] [d], mediante la realización plena del objetivo de cada gesto articulatorio, o bien, como sucede en la secuencia de [1] más fricativa, con la aparición de un breve cierre oclusivo en el paso del gesto del ápice a la lámina de la lengua ${ }^{19}$.

\section{Comentarios Finales}

A lo largo de estas páginas hemos visto que el debilitamiento de africada $/ \mathrm{t} \int /$, presenta un mismo patrón en las dos variantes de estudio. El acercamiento instrumental ha permitido constatar que en los contextos en los que aparentemente no ocurre el debilitamiento, el momento de oclusión de la africada representa una transición en tre los gestos articulatorios involucrados. Esto parece indicar que el proceso no está restringido a contextos específicos, sino que en dichas variedades del español la realización de la africada es una fricativa. La evidencia translingüística ha resultado iluminadora y ha ayudado a apuntalar esta hipótesis.

Esther Herrera Z.

El Colegio de México

${ }^{19} \mathrm{Al}$ respecto véase $\mathrm{H}$. M. Sussman y J. WESTbuRY, "The effects of antagonistic gestures on temporal and amplitude parameters of anticipatory labial coarticulation", Journal of Speech and Hearing Research, 24 (1981), 16-24, quienes proponen que hay grados de resistencia a la coarticulación dependiendo de los gestos articulatorios y de la cercanía entre ellos. 


\section{ANEXO}

Contexto de sílaba átona

fecha

dicha

techo

mucho

muchacho

escucho

leche

noche

la chavala

pecho

aprovecho

trapiche

de chamaquito

dicha

hacha

noches

cucho

michoacán

ficha

ocho

percha

parche

marcha

corcho

antorcha

escarcha

ancho

plancha

conchas

colcha

al chavalo

el chupón

\section{Contexto de sílaba tónica}

muchacho

cuchara

luchar

a la chinche

ochenta

puchero

escuchó

techó

cuchillo

aprovechó

una chaya

parchó

marchó

parché

parchar

un chango

un chivo

con chile

planchar

hinchado

pinché

un chino

rancheros

un chico

pinchó

se hinchó

al chicle

al chico 
